\title{
On the power-series expansion of a rational function
}

\author{
by \\ D. V. LEE (Nottingham)
}

Introduction. The problem of determining the formula for $P_{S}(n)$, the number of partitions of an integer into elements of a finite set $S$, that is, the number of solutions in non-negative integers, $h_{s_{1}}, \ldots, h_{s_{k}}$, of the equation

$$
h_{s_{1}} s_{1}+\ldots+h_{s_{k}} s_{k}=n,
$$

was solved in the nineteenth century (see Sylvester [4] and Glaisher [3] for detailed accounts). The solution is the coefficient of $x^{n}$ in

$$
\left[\left(1-x^{s_{1}}\right) \ldots\left(1-x^{s_{k}}\right)\right]^{-1}
$$

expressions for which they derived. Wright [5] indicated a simpler method by which to find part of the solution (at least in the case $s_{i}=i$ ).

The current paper gives a simple method by which the power-series expansion of a rational function may be derived. Lemma 1 is well known and gives the general form of the solution. Lemma 2 is also well known. See, for example, Andrews [1], Example 2, p. 98. Lemma 3 shows how the recurrence relation of Lemma 2 becomes of bounded degree in certain cases. The recurrence relation is then solved, and the solution is extended from these certain cases to all cases.

We then apply the result to investigate the growth of the difference $P_{S}(n)-P_{T}(n)$, where $S$ and $T$ are finite sets, and in particular when this difference is bounded. The differences $P_{S}^{(0)}(n)-P_{T}^{(0)}(n)$ and $P_{S}^{(1)}(n)-$ $P_{T}^{(1)}(n)$ are also considered, where $P_{S}^{(0)}$ (resp. $P_{S}^{(1)}$ ) denotes the number of partitions of $n$ into elements of $S$ with an even (resp. odd) number of parts.

Derivation of the power series of a rational function. Let

$$
g(x)=\prod_{i=1}^{k}\left(1-\alpha_{i} x\right)^{a_{i}} \quad \text { and } \quad f(x)=\prod_{i=k+1}^{l}\left(1-\alpha_{i} x\right)^{a_{i}^{\prime}}
$$

Research partially supported by SERC. 
be any polynomials with constant coefficient 1 , where the $\alpha_{i}$ are distinct and non-zero and the degree of $f(x)$ is less than that of $g(x)$. The power-series expansion of $f(x) / g(x)$ is derived (see Theorem 2).

\section{Lemma 1.}

$$
f(x) / g(x)=\sum_{n=0}^{\infty} \sum_{i=1}^{k} \sum_{j=0}^{a_{i}-1} b_{i, j} n^{j} \alpha_{i}^{n} x^{n},
$$

where the $b_{i, j}$ are constants and

$$
b_{i, a_{i}-1}=\frac{1}{\left(a_{i}-1\right) !} f\left(\alpha_{i}^{-1}\right) \prod_{\substack{\nu=1 \\ \nu \neq i}}^{k}\left(1-\alpha_{\nu} \alpha_{i}^{-1}\right)^{-a_{\nu}}=a_{i}\left(-\alpha_{i}\right)^{a_{i}} \frac{f\left(\alpha_{i}^{-1}\right)}{g^{\left(a_{i}\right)}\left(\alpha_{i}^{-1}\right)} .
$$

Proof. Write $f(x) / g(x)$ in partial fractions:

$$
f(x) / g(x)=\sum_{i=1}^{k} \sum_{t=1}^{a_{i}} A_{i, t} /\left(1-\alpha_{i} x\right)^{t}=\sum_{i=1}^{k} \sum_{t=1}^{a_{i}} A_{i, t} \sum_{n=0}^{\infty}\left(\begin{array}{c}
n+t-1 \\
t-1
\end{array}\right) \alpha_{i}^{n} x^{n} .
$$

Since $\left(\begin{array}{c}n+t-1 \\ t-1\end{array}\right)$ is a polynomial of degree $t-1$ in $n$, the form of $f(x) / g(x)$ is as given. Moreover,

$$
b_{i, a_{i}-1}=\frac{1}{\left(a_{i}-1\right) !} A_{i, a_{i}}
$$

as required. The second expression for $b_{i, a_{i}-1}$ follows by l'Hôpital's Rule.

Let

$$
f(x) / g(x)=\sum_{n=0}^{\infty} b(n) x^{n}
$$

LEMMA 2.

$$
n b(n)=\sum_{r=1}^{n}\left(\sum_{i=1}^{k} a_{i} \alpha_{i}^{r}-\sum_{i=k+1}^{l} a_{i}^{\prime} \alpha_{i}^{r}\right) b(n-r) .
$$

Proof.

$$
\frac{d}{d x} \log [f(x) / g(x)]=\sum_{i=1}^{k} \frac{a_{i} \alpha_{i}}{1-\alpha_{i} x}-\sum_{i=k+1}^{l} \frac{a_{i}^{\prime} \alpha_{i}}{1-\alpha_{i} x} .
$$

Hence

$$
\sum_{n=1}^{\infty} n b(n) x^{n-1}=\left\{\sum_{r=1}^{\infty}\left(\sum_{i=1}^{k} a_{i} \alpha_{i}^{r}-\sum_{i=k+1}^{l} a_{i}^{\prime} \alpha_{i}^{r}\right) x^{r-1}\right\}\left\{\sum_{s=0}^{\infty} b(s) x^{s}\right\},
$$

and the result follows by picking out the coefficient of $x^{n-1}$ on the right. 
Write $a_{i}=-a_{i}^{\prime}$ so that

$$
n b(n)=\sum_{r=1}^{n}\left(\sum_{i=1}^{l} a_{i} \alpha_{i}^{r}\right) b(n-r) .
$$

Consider the case where each $\alpha_{i}$ is a root of unity. In this case, write $\varrho_{i}$ instead of $\alpha_{i}$. Let $L$ be the least positive integer such that $\varrho_{i}^{L}=1$ for all $i$, and let $Z$ be any positive multiple of $L$.

LEMMA 3.

$$
(n+Z) b(n+Z)=n b(n)+\sum_{r=0}^{Z-1}\left(\sum_{i=1}^{l} a_{i} \varrho_{i}^{-r}\right) b(n+r) .
$$

Proof.

$$
\begin{aligned}
(n+Z) b(n+Z)= & \sum_{r=1}^{n+Z}\left(\sum_{i=1}^{l} a_{i} \varrho_{i}^{r}\right) b(n+Z-r) \\
= & \sum_{r=1}^{Z}\left(\sum_{i=1}^{l} a_{i} \varrho_{i}^{r}\right) b(n+Z-r) \\
& +\sum_{r=Z+1}^{n+Z}\left(\sum_{i=1}^{l} a_{i} \varrho_{i}^{r}\right) b(n+Z-r) \\
= & \sum_{s=0}^{Z-1}\left(\sum_{i=1}^{l} a_{i} \varrho_{i}^{-s}\right) b(n+s)+\sum_{t=1}^{n}\left(\sum_{i=1}^{l} a_{i} \varrho_{i}^{t}\right) b(n-t) \\
= & \sum_{s=0}^{Z-1}\left(\sum_{i=1}^{l} a_{i} \varrho_{i}^{-s}\right) b(n+s)+n b(n) .
\end{aligned}
$$

Let

$$
c_{j, n}=\sum_{i=1}^{k} b_{i, j} \varrho_{i}^{n}
$$

(where $b_{i, j}=0$ for $j \geq a_{i}$ ), so that

$$
b(n)=\sum_{j=0}^{m-1} c_{j, n} n^{j}, \quad \text { where } m=\max a_{i} .
$$

Put $b(n)=\sum_{j=0}^{m-1} c_{j, n} n^{j}$ in (1). Then

$$
\sum_{j=0}^{m-1} c_{j, n+Z}(n+Z)^{j+1}=\sum_{j=0}^{m-1} c_{j, n} n^{j+1}+\sum_{r=0}^{Z-1} \sum_{i=1}^{l} a_{i} \varrho_{i}^{-r} \sum_{j=0}^{m-1} c_{j, n+r}(n+r)^{j} .
$$


Now, $c_{j, n}$ is periodic in $n$ and hence bounded, so equate coefficients of $n^{q}$ in the above to obtain

(2) $\quad \sum_{j=q-1}^{m-1} c_{j, n+Z}\left(\begin{array}{c}j+1 \\ q\end{array}\right) Z^{j-q+1}$

$$
=c_{q-1, n}+\sum_{r=0}^{Z-1} \sum_{i=1}^{l} a_{i} \varrho_{i}^{-r} \sum_{j=q}^{m-1} c_{j, n+r}\left(\begin{array}{l}
j \\
q
\end{array}\right) r^{j-q}
$$

$\left(r^{j-q}=1\right.$ when $r=0$ and $\left.j=q\right)$. Note that

$$
b_{i, j}=\frac{1}{L} \sum_{n=1}^{L} \sum_{\nu=1}^{l} b_{\nu, j} \varrho_{\nu}^{n} \varrho_{i}^{-n}=\frac{1}{L} \sum_{n=1}^{L} c_{j, n} \varrho_{i}^{-n}, \quad 1 \leq i \leq k .
$$

Operate with $(1 / L) \sum_{n=1}^{L} \cdots \varrho_{p}^{-n}$ throughout $(2), 1 \leq p \leq k$, to get

$$
\sum_{j=q-1}^{m-1} b_{p, j}\left(\begin{array}{c}
j+1 \\
q
\end{array}\right) Z^{j-q+1}=b_{p, q-1}+\sum_{r=0}^{Z-1} \sum_{i=1}^{l} a_{i} \varrho_{i}^{-r} \sum_{j=q}^{m-1} b_{p, j} \varrho_{p}^{r}\left(\begin{array}{l}
j \\
q
\end{array}\right) r^{j-q} .
$$

Hence

(3) $\quad \sum_{j=q}^{a_{p}-1} b_{p, j}\left(\begin{array}{c}j+1 \\ q\end{array}\right) Z^{j-q+1}=\sum_{j=q}^{a_{p}-1} b_{p, j}\left(\begin{array}{l}j \\ q\end{array}\right) \sum_{r=0}^{Z-1} \sum_{i=1}^{l} a_{i} \varrho_{i}^{-r} \varrho_{p}^{r} r^{j-q}$.

Now drop the $p$ suffix. Observe $\sum_{r=0}^{Z-1}\left(\varrho_{i}^{-1} \varrho\right)^{r} r^{j-q}$ is the coefficient of $x^{j-q+1} /(j-q)$ ! in the power-series expansion of

$$
\begin{aligned}
x\left[1+\left(\varrho_{i}^{-1} \varrho\right) e^{x}\right. & \left.+\left(\varrho_{i}^{-1} \varrho\right)^{2} e^{2 x}+\ldots+\left(\varrho_{i}^{-1} \varrho\right)^{Z-1} e^{(Z-1) x}\right] \\
& =x\left(e^{Z x}-1\right) /\left(\varrho_{i}^{-1} \varrho e^{x}-1\right)=\frac{x}{\varrho_{i}^{-1} \varrho e^{x}-1} \sum_{s=1}^{\infty}(Z x)^{s} / s ! .
\end{aligned}
$$

Hence $\sum_{r=0}^{Z-1}\left(\varrho_{i}^{-1} \varrho\right)^{r} r^{j-q}$ is given by a polynomial in $Z$ whose coefficient of $Z$ is the coefficient of $x^{j-q} /(j-q)$ ! in the expansion of $x /\left(\varrho_{i}^{-1} \varrho e^{x}-1\right)$. Call this number $\gamma_{i, j-q}$. Since (3) holds for infinitely many values of $Z$, the coefficient of $Z$ on the left-hand side of the equation equals that on the right-hand side. This gives

$$
b_{q}(q+1)=\sum_{j=q}^{a-1} b_{j}\left(\begin{array}{l}
j \\
q
\end{array}\right) \sum_{i=1}^{l} a_{i} \gamma_{i, j-q} .
$$

Note that $\gamma_{i 0}$ is 1 when $i=p$ and zero otherwise. Hence

$$
(a-1-q) b_{q}=\sum_{j=q+1}^{a-1} b_{j}\left(\begin{array}{l}
j \\
q
\end{array}\right) d_{j-q}, \quad \text { where } d_{j-q}=-\sum_{i=1}^{l} a_{i} \gamma_{i, j-q} .
$$


Power-series expansion

233

Let

$$
b_{j}=\left(\begin{array}{c}
a-1 \\
a-1-j
\end{array}\right) u_{j}
$$

so that

$$
\begin{aligned}
(a-1-q) b_{q} & =\sum_{j=q+1}^{a-1}\left(\begin{array}{l}
j \\
q
\end{array}\right)\left(\begin{array}{c}
a-1 \\
a-1-j
\end{array}\right) u_{j} d_{j-q} \\
& =\sum_{j=q+1}^{a-1}\left(\begin{array}{c}
a-1 \\
a-1-q
\end{array}\right)\left(\begin{array}{c}
a-1-q \\
j-q
\end{array}\right) u_{j} d_{j-q},
\end{aligned}
$$

so

$$
u_{q}=\frac{1}{a-1-q} \sum_{j=q+1}^{a-1}\left(\begin{array}{c}
a-1-q \\
j-q
\end{array}\right) u_{j} d_{j-q} \quad \text { for } q<a-1 .
$$

Lemma 4. Let $j<a-1$. Then

(5)

$$
\begin{aligned}
& u_{j} / u_{a-1} \\
& =\sum_{\substack{v, n_{1}, \ldots, n_{v}>0 \\
n_{1}+\ldots+n_{v}=a-1-j}} \frac{1}{a-1-j}\left(\begin{array}{c}
a-1-j \\
n_{1}
\end{array}\right) \frac{1}{a-1-j-n_{1}}\left(\begin{array}{c}
a-1-j-n_{1} \\
n_{2}
\end{array}\right) \\
& \cdots \frac{1}{a-1-j-\sum_{s=1}^{t} n_{s}}\left(\begin{array}{c}
a-1-j-\sum_{s=1}^{t} n_{s} \\
n_{s+1}
\end{array}\right) \ldots \frac{1}{n_{v}}\left(\begin{array}{l}
n_{v} \\
n_{v}
\end{array}\right) \prod_{s=1}^{v} d_{n_{s}} .
\end{aligned}
$$

Proof. The formula is correct for $j=a-2$; the right-hand side is just $d_{1}$, in agreement with (4). Assume it is correct for all values of $j$ with $q<j<a-1$. Then

$$
\begin{aligned}
& u_{q} / u_{a-1} \\
& =\frac{1}{a-1-q}\left(\sum _ { j = q + 1 } ^ { a - 2 } \left\{\left(\begin{array}{c}
a-1-q \\
j-q
\end{array}\right) \sum_{\substack{v, n_{1}, \ldots, n_{v}>0 \\
n_{1}+\ldots+n_{v}=a-1-j}} \frac{1}{a-1-j}\left(\begin{array}{c}
a-1-j \\
n_{1}
\end{array}\right)\right.\right. \\
& \left.\left.\ldots \frac{1}{n_{v}}\left(\begin{array}{l}
n_{v} \\
n_{v}
\end{array}\right)\left[\prod_{s=1}^{v} d_{n_{s}}\right] d_{j-q}\right\}+d_{a-1-q}\right) \\
& =\left(\sum_{j=q+1}^{a-2} \sum_{\substack{v, n_{1}, \ldots, n_{v}>0 \\
(j-q)+n_{1}+\ldots+n_{v}=a-1-q}} \frac{1}{a-1-q}\left(\begin{array}{c}
a-1-q \\
j-q
\end{array}\right)\right. \\
& \times \frac{1}{a-1-q-(j-q)}\left(\begin{array}{c}
a-1-q-(j-q) \\
n_{1}
\end{array}\right) \\
& \left.\ldots \frac{1}{n_{v}}\left(\begin{array}{l}
n_{v} \\
n_{v}
\end{array}\right) d_{j-q} \prod_{s=1}^{v} d_{n_{s}}\right)+\frac{d_{a-1-q}}{a-1-q}
\end{aligned}
$$




$$
\begin{aligned}
= & \sum_{\substack{v, n_{0}, \ldots, n_{v}>0 \\
n_{0}+n_{1}+\ldots+n_{v}=a-1-q}} \frac{1}{a-1-q}\left(\begin{array}{c}
a-1-q \\
n_{0}
\end{array}\right) \\
& \left.\times \frac{1}{a-1-q-n_{0}}\left(\begin{array}{c}
a-1-q-n_{0} \\
n_{1}
\end{array}\right) \ldots \frac{1}{n_{v}}\left(\begin{array}{l}
n_{v} \\
n_{v}
\end{array}\right) \prod_{s=0}^{v} d_{n_{s}}\right)+\frac{d_{a-1-q}}{a-1-q} \\
= & \sum_{\substack{n_{0}, \ldots, n_{v}>0, v \geq 0 \\
n_{0}+n_{1}+\ldots+n_{v}=a-1-q}} \frac{1}{a-1-q}\left(\begin{array}{c}
a-1-q \\
n_{0}
\end{array}\right) \ldots \frac{1}{n_{v}}\left(\begin{array}{c}
n_{v} \\
n_{v}
\end{array}\right) \prod_{s=0}^{v} d_{n_{s}},
\end{aligned}
$$

and the result follows by induction.

Lemma 5.

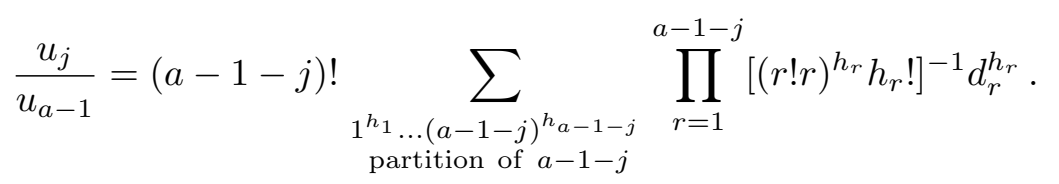

Proof. The product of the binomial coefficients in a term of (5) is

$$
\left(\begin{array}{c}
a-1-j \\
n_{1}, \ldots, n_{v}
\end{array}\right)=\frac{(a-1-j) !}{n_{1} ! \ldots n_{v} !} .
$$

It will suffice to show that for a given partition $1^{h_{1}} \ldots w^{h_{w}}$ of $w$,

$$
\sum \frac{1}{w_{1}\left(w_{1}+w_{2}\right)\left(w_{1}+w_{2}+w_{3}\right) \ldots(w)}=\frac{1}{\prod_{g=1}^{w} g^{h_{g} h_{g} !}},
$$

where $w_{1}, w_{2}, \ldots, w_{j}$ are the components of the partition, $j=\sum h_{g}$, and the summation on the left-hand side extends over all decompositions of $w$ with these components.

This is true for $w=1$ and in general for the partition $w=w^{1}$. Suppose it is true for all partitions of $y$ with $y<w$. Let $1^{h_{1}} \ldots w^{h_{w}}$ be a partition of $w$ with $h_{w}=0$. Then

$$
\begin{aligned}
\sum \frac{1}{w_{1}\left(w_{1}+w_{2}\right) \ldots(w)} & =\frac{1}{w} \sum_{\substack{w_{j}=1 \\
h_{w_{j}} \neq 0}}^{w-1} \sum \frac{1}{w_{1}\left(w_{1}+w_{2}\right) \ldots\left(w-w_{j}\right)} \\
& =\frac{1}{w} \sum_{\substack{w_{j}=1 \\
h_{w_{j}} \neq 0}}^{w-1}\left\{\left(\prod_{\substack{g=1 \\
g \neq w_{j}}}^{w} g^{h_{g}} h_{g} !\right) w_{j}^{h_{w_{j}}-1}\left(h_{w_{j}}-1\right) !\right\}^{-1} \\
& =\frac{1}{w} \sum_{\substack{w_{j}=1 \\
h_{w_{j}} \neq 0}}^{w-1} \frac{w_{j} h_{w_{j}}}{\prod_{g=1}^{w} g^{h_{g}} h_{g} !}=\frac{1}{\prod_{g=1}^{w} g^{h_{g}} h_{g} !}
\end{aligned}
$$


since

The result follows.

$$
\sum_{\substack{w_{j}=1 \\ h_{w_{j}} \neq 0}}^{w-1} w_{j} h_{w_{j}}=w
$$

Theorem 1.

$$
\begin{aligned}
b(n)=\sum_{i=1}^{k}\{ & \left.f\left(\varrho_{i}^{-1}\right) / \prod_{\substack{\nu=1 \\
\nu \neq i}}^{k}\left(1-\varrho_{\nu} \varrho_{i}^{-1}\right)^{a_{\nu}}\right\} \\
& \times \sum_{j=0}^{a_{i}-1} \frac{1}{j !} \sum_{\substack{h_{1} \ldots \ldots\left(a_{i}-1-j\right)^{h_{a}-1-j} \\
\text { partition of } a_{i}-1-j}}\left\{\prod_{r=1}^{a_{i}-1-j} \frac{d_{r}^{h_{r}}}{(r ! r)^{h_{r}} h_{r} !}\right\} n^{j} \varrho_{i}^{n},
\end{aligned}
$$

where $d_{r}$ is the coefficient of $x^{r} / r$ ! in the expansion of

$$
\sum_{\nu=1}^{l} \frac{a_{\nu} x}{1-\varrho_{\nu}^{-1} \varrho_{i} e^{x}}=x y \frac{h^{\prime}(y)}{h(y)},
$$

where $y=\left(\varrho_{i} e^{x}\right)^{-1}$ and $h(x)=[f(x) / g(x)]^{-1}$.

Proof. The form of $b(n)$ is immediate from Lemmas 1 and 5 . Note that

$$
\begin{aligned}
x y \frac{h^{\prime}(y)}{h(y)} & =x y \sum_{\nu=1}^{l} a_{\nu} \frac{d}{d y} \log \left(1-\varrho_{\nu} y\right)=x y \sum_{\nu=1}^{l}-\frac{a_{\nu} \varrho_{\nu}}{1-\varrho_{\nu} y} \\
& =x \sum_{\nu=1}^{l} \frac{a_{\nu}}{1-\varrho_{\nu}^{-1} y^{-1}}
\end{aligned}
$$

as required.

TheOrem 2. Let $a(n)$ be the coefficient of $x^{n}$ in the expansion of

$$
f(x) / g(x)=\left\{\prod_{i=k+1}^{l}\left(1-y_{i} x\right)^{a_{i}^{\prime}}\right\} /\left\{\prod_{i=1}^{k}\left(1-y_{i} x\right)^{a_{i}}\right\} .
$$

Let

$$
\begin{aligned}
& b(n)=\sum_{i=1}^{k}\left\{f\left(y_{i}^{-1}\right) / \prod_{\substack{\nu=1 \\
\nu \neq i}}^{k}\left(1-y_{\nu} y_{i}^{-1}\right)^{a_{i}}\right\}
\end{aligned}
$$

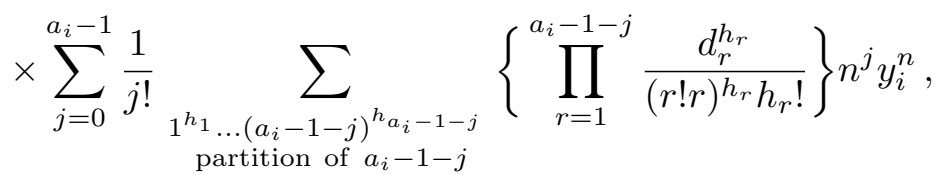


with $d_{r}$ the coefficient of $x^{r} / r !$ in the expansion of $\sum_{\nu=1}^{l}\left(a_{\nu} x /\left(1-y_{\nu}^{-1} y_{i} e^{x}\right)\right)$. Then $a(n)=b(n)$ holds whenever the $y_{i}$ are distinct and non-zero.

Proof. By Theorem 1, the result holds whenever the $y_{i}$ are distinct roots of unity. Observe $a(n)$ is the coefficient of $x^{n}$ in

$$
\left\{\prod_{i=k+1}^{l}\left(1-y_{i} x\right)^{a_{i}^{\prime}}\right\}\left\{\prod_{i=1}^{k}\left(\sum_{n=0}^{\infty}\left(\begin{array}{c}
n+a_{i}-1 \\
a_{i}-1
\end{array}\right) y_{i}^{n} x^{n}\right)\right\},
$$

so $a(n)$ is given by a polynomial, say $P\left(y_{1}, \ldots, y_{l}\right)$. For $a \neq 1$, let

$$
\frac{x}{1-a e^{x}}=\sum_{n=1}^{\infty} c_{n} \frac{x^{n}}{n !} \text {. }
$$

Then

so

$$
\left\{\sum_{n=1}^{\infty} c_{n} \frac{x^{n}}{n !}\right\}\left\{(a-1)+\sum_{n=1}^{\infty} a \frac{x^{n}}{n !}\right\}=-x,
$$

$$
\sum_{r=1}^{n-1} \frac{c_{r}}{r !} \frac{a}{(n-r) !}+\frac{c_{n}}{n !}(a-1)=0 \quad \text { for } n>1,
$$

and so

$$
c_{n}=\left\{\sum_{r=1}^{n-1} c_{r}\left(\begin{array}{l}
n \\
r
\end{array}\right)\right\} \frac{a}{1-a} \quad \text { for } n>1 .
$$

Since $c_{1}=1 /(1-a), c_{n}$ is a rational function of $a$ with denominator dividing $(1-a)^{n}$. Hence $a_{\nu} x /\left(1-y_{\nu}^{-1} y_{i} e^{x}\right)$ has as coefficient of $x^{n}$ a rational function of $y_{\nu}^{-1} y_{i}$ whose denominator is non-zero when $y_{\nu} \neq y_{i}$. When $y_{\nu}=y_{i}$ the coefficient is a constant. Therefore $d_{r}$ is given by a rational function in $y_{1}, \ldots, y_{l}$ whose denominator is non-zero when the $y_{i}$ are distinct. The same is true of $\prod_{\nu=1, \nu \neq i}^{k}\left(1-y_{\nu} y_{i}^{-1}\right)^{-a_{i}}$, and moreover $f\left(y_{i}^{-1}\right)$ is a rational function in $y_{1}, \ldots, y_{l}$ whose denominator is non-zero when $y_{i} \neq 0$. Hence $b(n)$ is a rational function in $y_{1}, \ldots, y_{l}$, say $Q\left(y_{1}, \ldots, y_{l}\right) / R\left(y_{1}, \ldots, y_{l}\right)$, where $R\left(y_{1}, \ldots, y_{l}\right)$ is non-zero for $y_{i}$ distinct non-zero. Therefore $P\left(y_{1}, \ldots, y_{l}\right)$ $\times R\left(y_{1}, \ldots, y_{l}\right)-Q\left(y_{1}, \ldots, y_{l}\right)=0$ holds whenever $y_{1}, \ldots, y_{l}$ are distinct roots of unity. That this holds for any distinct non-zero $y_{i}$ and hence $P\left(y_{1}, \ldots, y_{l}\right)=Q\left(y_{1}, \ldots, y_{l}\right) / R\left(y_{1}, \ldots, y_{l}\right)$ as required, now follows from Lemma 6.

Lemma 6. Let $F\left(X_{1}, \ldots, X_{n}\right)$ be a polynomial which vanishes whenever $X_{1}, \ldots, X_{n}$ are distinct roots of unity. Then $F\left(X_{1}, \ldots, X_{n}\right) \equiv 0$.

Proof. The case $n=1$ is clear, since a non-zero polynomial in one variable has only finitely many zeros. Suppose it is true for $1 \leq n \leq k-1$. Set $X_{1}, \ldots, X_{k-1}$ equal to distinct roots of unity, $\varrho_{1}, \ldots, \varrho_{k-1}$. Then $F\left(\varrho_{1}, \ldots, \varrho_{k-1}, X_{k}\right)$ is a polynomial in one variable with infinitely many 
zeros, so is identically zero. Thus the coefficient of each power of $X_{k}$ is zero. But this coefficient is a polynomial in $X_{1}, \ldots, X_{k-1}$ evaluated at any set of distinct roots of unity, so by the induction hypothesis this polynomial is identically zero. The result follows.

An application. Let $S$ and $T$ be finite multisets of positive integers. Suppose $P_{S}(n)-P_{T}(n)$ is bounded in $n$. Define an equivalence relation by $S \sim T$ when this holds. We attempt to characterise this relation. By Theorem 2, we have

$$
P_{S}(n)=\sum_{j=0}^{k-1} a_{j, n} n^{j}, \quad P_{T}(n)=\sum_{j=0}^{l-1} b_{j, n} n^{j},
$$

where $k=|S|, l=|T|$ and $a_{j, n}$ depends on $n \bmod \operatorname{lcm}\{S\}$ and $b_{j, n}$ depends on $n \bmod \operatorname{lcm}\{T\}$. Let $Z=\operatorname{lcm}\{S \cup T\}$. Then

$$
P_{S}(Z m+c)-P_{T}(Z m+c)=\sum_{j=0}^{\max (k, l)-1}\left(a_{j, c}-b_{j, c}\right)(Z m+c)^{j} .
$$

This being bounded in $m$ requires $a_{j, c}=b_{j, c}$ for all $j>0$. Put

$$
a_{j, n}=\sum_{\varrho} A_{\varrho, j} \varrho^{n} \quad \text { and } \quad b_{j, n}=\sum_{\varrho} B_{\varrho, j} \varrho^{n}
$$

where the sums are over all roots of unity of degree dividing $Z$. Hence

$$
\sum_{\varrho}\left(A_{\varrho, j}-B_{\varrho, j}\right) \varrho^{n}=0 \quad \text { for } j>0 .
$$

These equations for the $A_{\varrho, j}-B_{\varrho, j}$ are linearly independent for $1 \leq n \leq Z$ since the $\varrho$ are distinct (Vandermonde determinant). Therefore $A_{\varrho, j}=B_{\varrho, j}$ for all $\varrho$ and for all $j>0$. By Theorem 2,

$$
A_{\varrho, j}=\left\{\prod_{\substack{s \in S \\ d \mid s}} s \prod_{\substack{s \in S \\ d \nmid s}}\left(1-\varrho^{-s}\right)\right\}^{-1} \frac{1}{j !} \sum_{\substack{\text { partitions of } \\ N_{d}(S)-1-j}} \prod_{r=1}^{N_{d}(S)-1-j} \frac{d_{S, r}^{h_{r}}}{(r ! r)^{h_{r} h_{r} !}},
$$

where $\varrho$ is a primitive $d$ th root of unity, $N_{d}(S)$ elements of $S$ are divisible by $d$, and $d_{S, r}$ is the coefficient of $x^{r} / r !$ in $\sum_{s \in S}\left(s x /\left(1-\varrho^{s} e^{s x}\right)\right)$, so

$$
d_{S, r}=\sum_{a=0}^{d-1} d_{r}^{(a)} \sum_{s \equiv a(\bmod d)} s^{r}
$$

where $d_{r}^{(a)}$ is the coefficient of $x^{r} / r$ ! in $x /\left(1-\varrho^{a} e^{x}\right)$.

THEOREM 3. $S \sim T$ if and only if

(i) $N_{d}(S)=N_{d}(T)$ whenever $\max \left(N_{d}(S), N_{d}(T)\right) \geq 2$, 
(ii) $d_{S, r}=d_{T, r}$ for all $r$ with $1 \leq r \leq N_{d}-2$ for all $d$ with $N_{d}=N_{d}(S)=$ $N_{d}(T) \geq 3$,

(iii) $\prod_{s \in S, d \mid s} s=\prod_{t \in T, d \mid t} t$ whenever $N_{d} \geq 2$,

(iv) $\prod_{s \in S, d \nmid s}\left(1-\varrho^{-s}\right)=\prod_{t \in T, d \nmid t}\left(1-\varrho^{-t}\right)$ whenever $N_{d} \geq 2$, $\varrho$ a primitive d-th root of unity.

Proof. Suppose $S \sim T$. Then

$$
A_{\varrho, N_{d}(S)-1}=\left\{\prod_{\substack{s \in S \\ d \mid s}} s \prod_{\substack{s \in S \\ d \nmid s}}\left(1-\varrho^{-s}\right)\left(N_{d}(S)-1\right) !\right\}^{-1} \neq 0,
$$

where $N_{d}(S) \geq 1$, and $A_{\varrho, j}=0$ for $j \geq N_{d}(S)$. Moreover,

$$
B_{\varrho, N_{d}(T)-1}=\left\{\prod_{\substack{t \in T \\ d \mid t}} t \prod_{\substack{t \in T \\ d \nmid t}}\left(1-\varrho^{-t}\right)\left(N_{d}(T)-1\right) !\right\}^{-1} \neq 0,
$$

where $N_{d}(T) \geq 1$, and $B_{\varrho, j}=0$ for $j \geq N_{d}(T)$. Hence $N_{d}(S)=N_{d}(T)$ unless $N_{d}(S)+N_{d}(T)=1$, and (i) follows. It then follows that

$$
\prod_{s, d \mid s} s \prod_{s, d \nmid s}\left(1-\varrho^{-s}\right)=\prod_{t, d \mid t} t \prod_{t, d \nmid t}\left(1-\varrho^{-t}\right)
$$

when $N_{d} \geq 2$. Now suppose $N_{d} \geq 3$ and $1 \leq r \leq N_{d}-2$. Note

$$
A_{\varrho, N_{d}(S)-2}=\left\{\prod s \prod\left(1-\varrho^{-s}\right)\left(N_{d}-2\right) !\right\}^{-1} d_{S, 1}
$$

and

$$
B_{\varrho, N_{d}(T)-2}=\left\{\prod t \prod\left(1-\varrho^{-t}\right)\left(N_{d}-2\right) !\right\}^{-1} d_{T, 1},
$$

so that $d_{S, 1}=d_{T, 1}$. Suppose that $d_{S, g}=d_{T, g}$ for all $g$ with $1 \leq g<r$. Then

$$
A_{\varrho, N_{d}(S)-1-r}=B_{\varrho, N_{d}(T)-1-r}
$$

gives

$$
\left\{\prod s \prod\left(1-\varrho^{-s}\right)\left(N_{d}(S)-1-r\right) !\right\}^{-1}\left(\frac{d_{S, r}}{r ! r}-\frac{d_{T, r}}{r ! r}\right)=0,
$$

and so by induction (ii) holds. To prove (iii) and (iv) we introduce $N_{d, a}(S)$, the number of elements of $S$ such that $(s, d)=a$, where $a \mid d$.

LEMMA 7.

$$
N_{d, a}(S)=\sum_{m \mid d / a} \mu(m) N_{a m}(S) .
$$
$l \mid d$,

Proof. Fix $d$. Let $f(a)=N_{d, d / a}(S)$, and $g(a)=N_{d / a}(S)$. Then for

$$
\sum_{a \mid l} f(a)=\#\{s: d / l \mid(s, d)\}=N_{d / l}(S)=g(l) .
$$


Hence

$$
f\left(\frac{d}{a}\right)=\sum_{m \mid d / a} \mu(m) g\left(\frac{d}{a m}\right),
$$

as required. Since $a m \mid d$, we have $N_{a m}(S) \geq N_{d}(S)$ and hence $N_{a m}(S) \geq 2$, whence $N_{a m}(S)=N_{a m}(T)$. Thus $N_{d, a}(S)=N_{d, a}(T)$. Hence

$$
\frac{\prod_{s, d \nmid s}\left(1-\varrho^{-s}\right)}{\prod_{t, d \nmid t}\left(1-\varrho^{-t}\right)}=\prod_{\substack{a \mid d \\ a<d}}\left\{\frac{\prod_{(s, d)=a}\left(1-\varrho^{-s}\right)}{\prod_{(t, d)=a}\left(1-\varrho^{-t}\right)}\right\},
$$

where, for each $a$, the factor on the right-hand side has the same number of factors in the numerator as in the denominator. For fixed $a$, the numbers $1-\varrho^{-s}$ and $1-\varrho^{-t}$ are associates, and so $\prod_{s, d \nmid s}\left(1-\varrho^{-s}\right) / \prod_{t, d \nmid t}\left(1-\varrho^{-t}\right)$ is a unit. It is also positive and rational, so it is equal to unity. Now (iii) and (iv) follow. Conversely, if (i)-(iv) hold, then

$$
\begin{aligned}
& P_{S}(n)-P_{T}(n)=\sum_{\substack{N_{d} \geq 2 \\
d \mid s}}\left\{\prod_{\substack{s \\
d \nmid s}} s \prod_{\left.\left.d-\varrho^{-s}\right)\right\}^{-1}}(1-\right. \\
& \times\left(\frac{d_{S, N_{d}-1}}{\left(N_{d}-1\right) !\left(N_{d}-1\right)}-\frac{d_{T, N_{d}-1}}{\left(N_{d}-1\right) !\left(N_{d}-1\right)}\right) \varrho^{n} \\
& +\ldots+\sum_{\substack{\varrho \\
N_{d}(S)=1}}\left\{\prod_{\substack{s \\
d \mid s}} s \prod_{\substack{s \\
d \nmid s}}\left(1-\varrho^{-s}\right)\right\}^{-1} \varrho^{n} \\
& -\sum_{\substack{\varrho \\
N_{d}(T)=1}}\left\{\prod_{\substack{t \\
d \mid t}} t \prod_{\substack{t \\
d \nmid t}}\left(1-\varrho^{-t}\right)\right\}^{-1} \varrho^{n},
\end{aligned}
$$

which is periodic in $n$ with period $Z$.

The condition $\prod\left(1-\varrho^{-s}\right)=\prod\left(1-\varrho^{-t}\right)$. Let $\sigma_{a}=\sigma_{a}^{(0)}(d)=\#\{s \in$ $S: s \in a(\bmod d)\}-\#\{t \in T: t \in a(\bmod d)\}$, and let $S_{0}=\{s \in S: d \nmid s\}$.

LEMMA 8. The conditions of (iv) in association with condition (i) and $N_{d, a}(S)=N_{d, a}(T)$ hold if

(a) $\sigma_{a}+\sigma_{d-a}=0$ for all $a$,

(b) $\sum_{a=1}^{[d / 2]} \sigma_{a}$ is even and $\sum_{a=1}^{[d / 2]} a \sigma_{a} \equiv 0(\bmod d)$

for all $d$ with $N_{d} \geq 2$.

Proof.

$$
\frac{\prod_{s \in S_{0}}\left(1-\varrho^{-s}\right)}{\prod_{t \in T_{0}}\left(1-\varrho^{-t}\right)}=\prod_{a=1}^{d-1}\left(1-\varrho^{-a}\right)^{\sigma_{a}}=\prod_{a=1}^{[d / 2]}\left\{\frac{1-\varrho^{-a}}{1-\varrho^{a}}\right\}^{\sigma_{a}}
$$


by (a). Hence

$$
\frac{\prod_{s \in S_{0}}\left(1-\varrho^{-s}\right)}{\prod_{t \in T_{0}}\left(1-\varrho^{-t}\right)}=\prod_{a=1}^{[d / 2]}\left(-\varrho^{-a}\right)^{\sigma_{a}}=1 .
$$

CONJeCture 1. The conditions of this lemma are necessary.

We observe that, given (a), we cannot have $d$ even, $\sum_{a=1}^{d / 2} a \sigma_{a} \equiv d / 2$ $(\bmod d)$ and $\sum_{a=1}^{d / 2} \sigma_{a}$ odd by showing that these conditions are inconsistent with $\frac{1}{2} d \mid \sum_{a=1}^{[d / 4]} a \sigma_{a}(d / 2)$ and $\sum_{a=1}^{[d / 4]} \sigma_{a}(d / 2)$ even. The required result then follows by induction. We have

$$
\begin{aligned}
\sum_{a=1}^{[d / 4]} \sigma_{a}(d / 2) & =\sum_{a=1}^{[d / 4]}\left\{\sigma_{a}(d)+\sigma_{a+d / 2}(d)\right\} \\
& =\sum_{a=1}^{[d / 4]}\left\{\sigma_{a}(d)-\sigma_{d / 2-a}(d)\right\} \equiv \sum_{\substack{a=1 \\
a \neq d / 4}}^{d / 2} \sigma_{a}(d)(\bmod 2) .
\end{aligned}
$$

This is a contradiction unless $4 \mid d$, in which case $\sigma_{d / 4}(d)$ is odd. If $4 \mid d$, then

$$
0 \equiv \sum_{a=1}^{d / 4-1} a\left\{\sigma_{a}(d)-\sigma_{d / 2-a}(d)\right\} \equiv \sum_{\substack{a=1 \\ a \neq d / 4}}^{d / 2-1} a \sigma_{a}(d)(\bmod d / 2),
$$

and hence $\frac{1}{2} d \mid \frac{1}{4} d \sigma_{d / 4}(d)$, which is a contradiction. Thus (b) is necessary given (a).

If we let $\tau_{a}=\sigma_{a}+\sigma_{d-a}$, then expressing condition (iv) for $d^{\prime}, d^{\prime} \mid d$, in terms of fundamental units for $\mathbb{Z}\left[\varrho^{d / d^{\prime}}\right]$ gives $\frac{1}{2} \phi\left(d^{\prime}\right)-1$ homogeneous linear equations in the $\tau_{a}$ when $d^{\prime} \neq 1,2$. Further such equations follow from $N_{d, a}(S)=N_{d, a}(T)$ for each $a \mid d, a<d$. The total number of equations is $[d / 2]$, so Conjecture 1 is equivalent to the independence of these equations. The conjecture can be proved by this method when $d$ is a prime power, owing to the relatively simple nature of the fundamental units in this case.

The condition $d_{S, r}=d_{T, r}$. We attempt to simplify the condition (ii). Let

$$
d_{r}^{(a)}=f_{r}\left(\varrho^{a}\right) \quad \text { and } \quad \sigma_{a}=\sigma_{a}^{(r)}=\sum_{s \equiv a(\bmod d)} s^{r}-\sum_{t \equiv a(\bmod d)} t^{r},
$$

so by (6) condition (ii) says

$$
\sum_{a=0}^{d-1} f_{r}\left(\varrho^{a}\right) \sigma_{a}=0
$$


for $\varrho$ a primitive $d$ th root of unity. However, since $N_{l} \geq N_{d}$, a similar equation holds for $\varrho$ a primitive $l$ th root of unity, where $l \mid d$, namely

which is to say

$$
\sum_{b=0}^{l-1} f_{r}\left(\varrho^{b}\right) \sum_{\substack{a=0 \\ a \equiv b(\bmod l)}}^{d-1} \sigma_{a}=0,
$$

$$
\sum_{a=0}^{d-1} f_{r}\left(\varrho^{a}\right) \sigma_{a}=0 .
$$

Hence this equation holds for all $\varrho$ such that $\varrho^{d}=1$.

Lemma 9. Let $h_{r}(\mu)=f_{r}(\mu)-f_{r}(1)$. Then

$$
h_{r}(\mu)=(-1)^{r} h_{r}\left(\mu^{-1}\right) .
$$

Pro of. It suffices to show

$$
\frac{x}{1-\mu e^{x}}-\frac{x}{1-e^{x}}=\frac{-x}{1-\mu^{-1} e^{-x}}-\frac{-x}{1-e^{-x}},
$$

which is easily verified. Actually, $f_{r}(\mu)=(-1)^{r} f_{r}\left(\mu^{-1}\right)$ for $r>1$.

Corollary. The equations (7) hold if

$$
\sigma_{a}+(-1)^{r} \sigma_{d-a}=0
$$

for all $a$, and also $\sum_{a=0}^{d-1} \sigma_{a}=0$ when $r=1$.

Proof. Equation (7) with $\varrho=1$ is vacuous if $f_{r}(1)=0$, and is equivalent to $\sum_{a=0}^{d-1} \sigma_{a}=0$ if $f_{r}(1) \neq 0$. Now $f_{r}(1) \neq 0$ if and only if $r=1$ or $r$ is even, by the well-known property of Bernoulli numbers. If $r$ is even, (9) gives

$$
\sum_{a=0}^{d-1} \sigma_{a}=\frac{1}{2} \sum_{a=0}^{d-1}\left(\sigma_{a}+\sigma_{d-a}\right)=0
$$

as required. Subtracting equation (7) with $\varrho=1$ from equation (7) with $\varrho=\mu \neq 1, \mu^{d}=1$, yields

or

$$
\sum_{a=0}^{d-1}\left[f_{r}\left(\mu^{a}\right)-f_{r}(1)\right] \sigma_{a}=0
$$

$$
\sum_{a=0}^{d-1} h_{r}\left(\mu^{a}\right) \sigma_{a}=0 .
$$

Hence, by (8), equations (9) give a solution.

Conjecture 2. The equations (9) are necessary.

Alternatively, the matrix $\left(h_{r}\left(\mu^{i j}\right)\right)_{\substack{1 \leq i<d / 2 \\ 1 \leq j<d / 2}}, r$ odd, or $\left(h_{r}\left(\mu^{i j}\right)\right)_{\substack{1 \leq i \leq d / 2 \\ 1 \leq j \leq d / 2}}$, $r$ even, is non-singular, where $\mu$ is a primitive $d$ th root of unity. 
Instead of the bounds on $i$ given, we could take $i \in I$, where $I$ consists of $[(d-1) / 2]$ of the non-zero residues modulo $d$ such that $I \cap-I=\emptyset$, unless $r$ is even and $d$ is even, in which case $I$ should be replaced by $I \cup\{d / 2\}$.

The following two lemmas give some properties of $f_{r}(\mu)$ useful for computation.

Lemma 10.

$$
\sum_{\mu, \mu^{d}=1} f_{r}(\mu)=d^{r} f_{r}(1)
$$

and hence

(10) $\sum_{\substack{\text { primitive } d \text { th } \\ \text { root of } 1}} f_{r}(\mu)=\sum_{m \mid d} \mu(d / m) m^{r} f_{r}(1)=d^{r} \prod_{p \mid d}\left(1-p^{-r}\right) f_{r}(1)$.

Proof. This follows from

$$
\sum_{\mu, \mu^{d}=1} \frac{x}{1-\mu e^{x}}=\frac{d x}{1-e^{d x}}
$$

a consequence of the formula for $d_{r}$ in Theorem 2 .

LEMMA 11.

$$
f_{r}(\mu)=\sum_{k=0}^{r-1} \sum_{h=0}^{k} r\left(\begin{array}{c}
r \\
k-h
\end{array}\right)(-1)^{k-h} h^{r-1} \mu^{k} /(1-\mu)^{r}
$$

for $\mu \neq 1$.

(Corollary: $f_{r}(1)=\sum_{k=0}^{r-1} \sum_{h=0}^{k} r\left(\begin{array}{c}r \\ k-h\end{array}\right)(-1)^{h} h^{r-1} / 2^{r}\left(2^{r}-1\right)$ by $(10)$ with $d=2$.)

Proof. We have

$$
f_{r}(\mu)=\sum_{m=1}^{r-1} f_{m}(\mu)\left(\begin{array}{c}
r \\
m
\end{array}\right) \frac{\mu}{1-\mu} \quad \text { and } \quad f_{1}(\mu)=\frac{1}{1-\mu} .
$$

It follows easily by induction that there exist constants $a_{r, k}$ such that

$$
f_{r}(\mu)=\sum_{k=1}^{r-1} a_{r, k} \mu^{k} /(1-\mu)^{r}
$$

By (11),

$$
\begin{aligned}
\frac{\sum_{k=0}^{r-1} a_{r, k} \mu^{k}}{(1-\mu)^{r}} & =\sum_{m=1}^{r-1} \sum_{j=0}^{m-1} a_{m, j} \frac{\mu^{j}}{(1-\mu)^{r}}\left(\begin{array}{c}
r \\
m
\end{array}\right) \frac{\mu}{1-\mu} \\
& =\sum_{m=1}^{r-1} \sum_{j=0}^{m-1} a_{m, j}\left(\begin{array}{c}
r \\
m
\end{array}\right) \sum_{l=0}^{r-m-1}\left(\begin{array}{c}
r-m-1 \\
l
\end{array}\right)(-1)^{l} \mu^{j+l+1} /(1-\mu)^{r}
\end{aligned}
$$




$$
=\sum_{m=1}^{r-1} \sum_{k=1}^{r-1} \sum_{j=0}^{k-1} a_{m, j}\left(\begin{array}{c}
r \\
m
\end{array}\right)\left(\begin{array}{c}
r-1-m \\
k-1-j
\end{array}\right)(-1)^{k-j-1} \mu^{k} /(1-\mu)^{r} .
$$

Hence

$$
a_{r, k}=\sum_{m=1}^{r-1} \sum_{j=0}^{k-1}(-1)^{k-j-1}\left(\begin{array}{c}
r-1-m \\
k-1-j
\end{array}\right)\left(\begin{array}{c}
r \\
m
\end{array}\right) a_{m, j} \quad \text { and } \quad a_{1,0}=1 .
$$

We show by induction on $r$ that

$$
a_{r, k}=\sum_{h=0}^{k} r\left(\begin{array}{c}
r \\
k-h
\end{array}\right)(-1)^{k-h} h^{r-1} .
$$

This satisfies $a_{1,0}=1$. Suppose it is correct for all $m$ with $1 \leq m \leq r-1$.

Then

$$
\begin{aligned}
a_{r, k} & =\sum_{m=1}^{r-1} \sum_{j=0}^{k-1}(-1)^{k-j-1}\left(\begin{array}{c}
r-1-m \\
k-1-j
\end{array}\right)\left(\begin{array}{c}
r \\
m
\end{array}\right) a_{m, j} \\
& =\sum_{m=1}^{r-1} \sum_{j=0}^{k-1}(-1)^{k-j-1}\left(\begin{array}{c}
r-1-m \\
k-1-j
\end{array}\right)\left(\begin{array}{c}
r \\
m
\end{array}\right) \sum_{h=0}^{j} m\left(\begin{array}{c}
m \\
j-h
\end{array}\right)(-1)^{j-h} h^{m-1} \\
& =\sum_{h=0}^{k-1} \sum_{m=1}^{r-1} \sum_{j=h}^{k-1}(-1)^{k-h-1}\left(\begin{array}{c}
r-1-m \\
k-1-j
\end{array}\right)\left(\begin{array}{c}
m \\
j-h
\end{array}\right)\left(\begin{array}{c}
r \\
m
\end{array}\right) m h^{m-1} \\
& =\sum_{h=0}^{k-1} \sum_{m=1}^{r-1}(-1)^{k-h-1}\left(\begin{array}{c}
r-1 \\
k-1-h
\end{array}\right)\left(\begin{array}{c}
r \\
m
\end{array}\right) m h^{m-1}
\end{aligned}
$$

since $\sum_{j}\left(\begin{array}{c}r-1-m \\ k-1-j\end{array}\right)\left(\begin{array}{c}m \\ j-h\end{array}\right)$ is the coefficient of $x^{k-1-h}$ in $(1+x)^{r-1-m}(1+x)^{m}$. Now

so

$$
\begin{aligned}
\sum_{m=1}^{r-1}\left(\begin{array}{c}
r \\
m
\end{array}\right) m h^{m-1} & =\frac{d}{d h} \sum_{m=1}^{r-1}\left(\begin{array}{c}
r \\
m
\end{array}\right) h^{m} \\
& =\frac{d}{d h}\left[(h+1)^{r}-h^{r}\right]=r\left[(h+1)^{r-1}-h^{r-1}\right],
\end{aligned}
$$

$$
\begin{aligned}
a_{r, k} & =\sum_{h=0}^{k-1}(-1)^{k-1-h}\left(\begin{array}{c}
r-1 \\
k-1-h
\end{array}\right) r\left[(h+1)^{r-1}-h^{r-1}\right] \\
& =\sum_{h=0}^{k}(-1)^{k-h} r\left[\left(\begin{array}{c}
r-1 \\
k-1-h
\end{array}\right)+\left(\begin{array}{l}
r-1 \\
k-h
\end{array}\right)\right] h^{r-1} \\
& =\sum_{h=0}^{k}(-1)^{k-h} r\left(\begin{array}{c}
r \\
k-h
\end{array}\right) h^{r-1},
\end{aligned}
$$

as required. 
Note that it follows from (8) and $f_{r}(1)=0$ for odd $r>1$ that $a_{r, k}=$ $a_{r, r-k}$. If $g_{r}(\mu)=(1-\mu)^{r} f_{r}(\mu)$, then

$$
g_{r}(\mu)=(1-\mu)^{r-1-m}\left(\begin{array}{c}
r \\
m
\end{array}\right) \mu g_{m}(\mu),
$$

so that $g_{r}(1)=r g_{r-1}(1)$ and hence $g_{r}(1)=r$ !. Thus we see that $\sum_{k=0}^{r-1} a_{r, k}$ $=r !$.

The following is a generalisation of Lemma 10 .

LEMMA 12.

$$
c_{l}:=\sum_{\substack{\mu \\
\mu^{d}=1}} \mu^{-l} f_{r}(\mu)=\sum_{j=0}^{r}\left(\begin{array}{l}
r \\
j
\end{array}\right) f_{j}(1) d^{j} l^{r-j}
$$

for $1 \leq l \leq d-1$. Also $c_{l}=(-1)^{r} c_{d-l}$ for $1 \leq l \leq d-1$, and

$$
f_{r}(\mu)=\frac{1}{d}\left\{c_{0}+\mu c_{1}+\ldots+\mu^{d-1} c_{d-1}\right\}
$$

Proof. This is a consequence of

$$
\sum_{\substack{\mu \\ \mu^{d}=1}} \mu^{-l} \frac{x}{1-\mu e^{x}}=\frac{d x e^{l x}}{1-e^{d x}} \quad \text { for } \quad 0 \leq l \leq d-1 .
$$

For $\mu \neq 1$, the denominator of $f_{r}(\mu)$ divides $(1-\mu)^{r}$. For $r>1$, odd, $f_{r}(1)=0$, so if $p$, prime, divides the denominator of $c_{l}$ then $p \mid d$. The denominator of $f_{j}(1)$ is square-free for all $j$ and $f_{0}(1)=-1, f_{1}(1)=1 / 2$, so $c_{l}$ is an integer congruent to

$$
-l^{r} \bmod \begin{cases}\frac{1}{2} d, & 2 \mid d, \\ d, & 2 \nmid d .\end{cases}
$$

Note that the congruence is also valid for $r$ even and $r=1$, although $c_{l}$ may not be an integer.

We may also note that $f_{r}(\mu)$ is the analytic continuation of the power series $r \sum_{l=0}^{\infty} l^{r-1} \mu^{l}$ convergent for $|\mu|<1$. To see this, observe that

$$
\sum_{r} \frac{f_{r}(\mu)}{r !} x^{r}=x \sum_{m=0}^{\infty}\left(\mu e^{x}\right)^{m}=x \sum_{m=0}^{\infty} \sum_{j=0}^{\infty} \mu^{m} \frac{(m x)^{j}}{j !}
$$

for $\left|\mu e^{x}\right|<1$, and equate coefficients. It follows immediately from this comment that, for example,

$$
\sum_{\varrho, \varrho^{d}=1} f_{r}(\varrho \mu)=d^{r} f_{r}\left(\mu^{d}\right) .
$$




\section{The conjecture for small $d$}

\section{$d=1$ : Nothing to prove.}

$d=2$ : Something to prove when $r$ is even. The conjecture holds if and only if $h_{r}(-1) \neq 0$ for $r$ even, but by $(10) h_{r}(-1)=\left(2^{r}-2\right) f_{r}(1) \neq 0$ as required.

$d=3$ : We need $h_{r}(\varrho) \neq 0, \varrho^{2}+\varrho+1=0$. We have $h_{r}(\varrho)+h_{r}\left(\varrho^{2}\right)=$ $\left(3^{r}-3\right) f_{r}(1)$ and $h_{r}(\varrho)=(-1)^{r} h_{r}\left(\varrho^{2}\right)$, so for $r$ even $h_{r}(\varrho)=\frac{1}{2}\left(3^{r}-3\right) f_{r}(1)$ $\neq 0$. For $r>1$, odd, $\varrho^{2} f_{r}(\varrho)+\varrho f_{r}\left(\varrho^{2}\right) \equiv-1(\bmod 3)$ by Lemma 12 , so $h_{r}(\varrho)=f_{r}(\varrho) \neq 0$. For $r=1$, we have $h_{1}(\varrho)=(1-\varrho)^{-1}-\frac{1}{2} \neq 0$.

$d=4$ : We require $h_{r}(i) \neq 0$ when $r$ is odd, and

$$
\left|\begin{array}{cc}
h_{r}(i) & h_{r}(-1) \\
h_{r}(-1) & h_{r}(1)
\end{array}\right| \neq 0
$$

when $r$ is even. For $r$ even we require $h_{r}(-1) \neq 0$, since $h_{r}(1)=0$, and this holds as for $d=2$. For $r>1$ odd,

$-i f_{r}(i)+i f_{r}(-i)-f_{r}(-1)+f_{r}(1) \equiv-1(\bmod 2)$ and $f_{r}(1)=f_{r}(-1)=0$, so $h_{r}(i)=f_{r}(i) \neq 0$. Also $h_{1}(i) \neq 0$.

$d=5$ : We require $h_{r}(\varrho) h_{r}\left(\varrho^{4}\right)-h_{r}\left(\varrho^{2}\right)^{2} \neq 0$, or equivalently $(-1)^{r} h_{r}(\varrho)^{2}$ $-h_{r}\left(\varrho^{2}\right)^{2} \neq 0$. Suppose this is not so. Let $z_{i}=h_{r}\left(\varrho^{i}\right)$. For $r>1$, odd, we have $z_{1}= \pm i z_{2}$, and so $z_{1}=z_{2}=z_{3}=z_{4}=0$, since $i \notin \mathbb{Q}(\varrho)$. However, $\sum_{i=1}^{4} \mu^{-i} z_{i} \equiv-1(\bmod 5)$, so we have a contradiction. For $r$ even we have $z_{1}= \pm z_{2}$. If $z_{1}=-z_{2}$ then $f_{r}(\varrho)+f_{r}\left(\varrho^{2}\right)=2 f_{r}(1)$, and hence $c_{0}=5 f_{r}(1)$. But $c_{0}=5^{r} f_{r}(1)$ and $f_{r}(1) \neq 0$, which is a contradiction. If $z_{1}=z_{2}$, then $c_{1}=c_{2}=c_{3}=c_{4}$, so

$$
z_{1}=\frac{1}{5}\left(c_{0}-c_{1}\right)=-\frac{1}{5} \sum_{j=0}^{r-1}\left(\begin{array}{l}
r \\
j
\end{array}\right) f_{j}(1) 5^{j}=-\frac{1}{5} \sum_{j=1}^{r-1}\left(\begin{array}{l}
r \\
j
\end{array}\right) f_{j}(1)\left(5^{j}-1\right)
$$

since $\sum_{j=0}^{r-1}\left(\begin{array}{l}r \\ j\end{array}\right) f_{j}(1)=0$. By $(10)$, we have $z_{1}=\frac{1}{4}\left(5^{r}-1\right) f_{r}(1)$. We shall show that these two formulae for $z_{1}$ are incompatible. Suppose $2^{k} \| r$. If $2^{l} \| j$, then $2^{k-\min (k, l)} \mid\left(\begin{array}{l}r \\ j\end{array}\right)$ and $2^{l+2} \|\left(5^{j}-1\right)$. Hence the numerator of $\left(\begin{array}{l}r \\ j\end{array}\right) f_{j}(1)\left(5^{j}-1\right)$ is divisible by $2^{k-l-1+l+2}=2^{k+1}$. Hence $\frac{1}{4}\left(5^{r}-1\right) f_{r}(1)$ has numerator divisible by $2^{k+1}$, and so $2^{k+4} \mid\left(5^{r}-1\right)$, whence $2^{k+2} \mid r$, contradicting $2^{k} \| r$.

The case $r=1$ and $d=p$, an odd prime. For $\mu^{p}=1, \mu \neq 1$, we have

$$
2 h_{1}(\mu)=\frac{1+\mu}{1-\mu}
$$


Hence

$$
\sum_{a=1}^{p-1} \frac{1+\mu^{a}}{1-\mu^{a}} \sigma_{a}=0
$$

Using (8), write this as

$$
\sum_{\substack{a=1 \\ 1 \leq a^{*} \leq(p-1) / 2}}^{p-1} \frac{1+\mu^{a}}{1-\mu^{a}}\left(\sigma_{a}-\sigma_{p-a}\right)=0,
$$

where $a^{*}$ is the least positive integer such that $a a^{*} \equiv 1(\bmod p)$. We derive a condition equivalent to the condition that the numbers $\left(1+\mu^{a}\right) /\left(1-\mu^{a}\right)$ are linearly independent, which in turn is equivalent to the conjecture. Now

$$
\begin{aligned}
(1-\mu) \frac{1+\mu^{a}}{1-\mu^{a}} & =\left(1+\mu^{a}\right)\left(1+\mu^{a}+\mu^{2 a}+\ldots+\mu^{\left(a^{*}-1\right) a}\right) \\
& =1+2 \mu^{a}+2 \mu^{2 a}+\ldots+2 \mu^{\left(a^{*}-1\right) a}+\mu .
\end{aligned}
$$

Reduce the exponents in (12) modulo $p$ and replace $\mu^{p-1}$ by $-\left(\mu^{p-2}\right.$ $\left.+\mu^{p-3}+\ldots+1\right)$. The coefficient of $\mu^{0}$ (and of $\mu^{1}$ ) is now +1 or -1 according as there does not exist or there exists $r, 1 \leq r \leq a^{*}-1$, such that $r a \equiv-1(\bmod p)$. This condition on $r$ means $p-a^{*} \leq a^{*}-1$, so $a^{*} \geq \frac{1}{2}(p+1)$. Hence the coefficient of $\mu^{0}$ (and of $\mu^{1}$ ) is 1 . The coefficient of $\mu^{m}, 2 \leq m \leq p-2$, has a contribution +2 if there exists $r, 1 \leq r \leq a^{*}-1$, such that $a r \equiv m(\bmod p)$ and is zero otherwise $\left(\right.$ since $\left.a^{*} \leq \frac{1}{2}(p-1)\right)$. This condition on $r$ is equivalent to $\left[m a^{*}\right]_{p} \leq a^{*}-1$, where $[k]_{p}$ means the least non-negative residue of $k(\bmod p)$. Note that this condition is always false when $m=2$, since

$$
\left[2 a^{*}\right]_{p} \leq a^{*}-1 \Leftrightarrow a^{*} \geq \frac{1}{2}(p+1) .
$$

Note also that $\left[m a^{*}\right]_{p}+\left[(p+1-m) a^{*}\right]_{p} \equiv a^{*}(\bmod p)$ and so $\left[m a^{*}\right]_{p} \leq a^{*}-1$ if and only if $\left[(p+1-m) a^{*}\right]_{p} \leq a^{*}-1$. Therefore we need consider only $3 \leq m \leq \frac{1}{2}(p+1)$ and $m=0$.

When $a^{*}=1$ the coefficient is non-zero when $m=0$ and zero for $3 \leq$ $m \leq \frac{1}{2}(p+1)$, and when $a^{*}=2$ the coefficient is non-zero only when $m=\frac{1}{2}(p+1)$. Hence the $\frac{1}{2}(p-1)$ equations obtained by varying $m$ are linearly independent if and only if the $\frac{1}{2}(p-5)$ equations corresponding to $3 \leq m \leq \frac{1}{2}(p-1)$ are linearly independent. Halving the matrix of coefficients for these equations gives the following theorem.

THEOREM 4. The conjecture with $r=1$ and $d=p$, an odd prime, holds if and only if the matrix

$$
\left(a_{i j}\right)_{\substack{3 \leq i \leq(p-1) / 2 \\ 3 \leq j \leq(p-1) / 2}} \quad \text { where } a_{i j}= \begin{cases}1 & \text { when }[i j]_{p} \leq i-1 \\ 0 & \text { otherwise }\end{cases}
$$


is non-singular.

We shall show via Theorem 5 that this matrix is indeed non-singular. Obviously a similar analysis with $r>1$ is not so simple.

The case $d=p$, an odd prime. We choose the set $I$ mentioned after Conjecture 2 to be $\left\{1, g, g^{2}, \ldots, g^{(p-1) / 2-1}\right\}$, where $g$ is a primitive root modulo $p$. Let $l=\frac{1}{2}(p-1)$ and write $h_{i}$ for $h_{r}\left(\mu^{i}\right)$. Then the matrix of Conjecture 2 becomes

for $r$ even,

$$
\left(\begin{array}{ccccc}
h_{1} & h_{g} & h_{g^{2}} & \cdots & h_{g^{l-1}} \\
h_{g} & h_{g^{2}} & h_{g^{3}} & \cdots & h_{1} \\
h_{g^{2}} & h_{g^{3}} & h_{g^{4}} & \cdots & h_{g} \\
\vdots & \vdots & \vdots & & \vdots \\
h_{g^{l-1}} & h_{1} & h_{g} & \cdots & h_{g^{-2}}
\end{array}\right)
$$

$$
\left(\begin{array}{ccccc}
h_{1} & h_{g} & h_{g^{2}} & \cdots & h_{g^{l-1}} \\
h_{g} & h_{g^{2}} & h_{g^{3}} & \cdots & -h_{1} \\
h_{g^{2}} & h_{g^{3}} & h_{g^{4}} & \cdots & -h_{g} \\
\vdots & \vdots & \vdots & & \vdots \\
h_{g^{l-1}} & -h_{1} & -h_{g} & \cdots & -h_{g^{-2}}
\end{array}\right)
$$

for $r$ odd, since $h_{i}=(-1)^{r} h_{-i}$ by Lemma 9 . The determinant of the first matrix is

$$
(-1)^{[l / 2]} \prod_{\eta, \eta^{l}=1}\left(\sum_{i=0}^{l-1} \eta^{i} h_{g^{i}}\right)
$$

and that of the second is

$$
(-1)^{l-1+[l / 2]} \prod_{\eta, \eta^{l}=-1}\left(\sum_{i=0}^{l-1} \eta^{i} h_{g^{i}}\right) .
$$

For $\omega$ a primitive $k$ th root of unity, $k \mid p-1$, let $\left(\frac{a}{p}\right)_{k}=\omega^{i}$, where $a \equiv g^{i}$ $(\bmod p)$. Then the Conjecture 2 holds if and only if

$$
\sum_{\substack{i=0 \\ a \equiv g^{i}}}^{l-1}\left(\frac{a}{p}\right)_{k} h_{a} \neq 0
$$

for all such $k$ with $k \nmid \frac{1}{2}(p-1)$ for $r$ odd and $k \mid \frac{1}{2}(p-1)$ for $r$ even. Noting that

$$
\left(\frac{-a}{p}\right)_{k} h_{-a}=\left(\frac{-1}{p}\right)_{k}(-1)^{r}\left(\frac{a}{p}\right)_{k} h_{a}=\left(\frac{a}{p}\right)_{k} h_{a},
$$

we obtain the following. 
if

TheOREM 5. The conjecture with $d=p$, an odd prime, holds if and only $\sum_{a=1}^{p-1}\left(\frac{a}{p}\right)_{k} h_{a} \neq 0 \quad$ for all $k \mid p-1$ such that $\quad \begin{cases}k \nmid \frac{1}{2}(p-1) & \text { for } r \text { odd }, \\ k \mid \frac{1}{2}(p-1) & \text { for } r \text { even. }\end{cases}$

In particular, when $r=1$, the matrix of Theorem 4 is non-singular if and only if

$$
\sum_{\substack{a=1 \\[m a]_{p} \leq a-1}}^{p-1}\left(\frac{a}{p}\right)_{k} \neq 0 \quad \text { for some } m(k), 2 \leq m \leq \frac{1}{2}(p-1),
$$

for all $k$ with $p \equiv k+1(\bmod 2 k)$.

Setting $m=2$ and using the following result proves Conjecture 2 for $d=p$ and $r=1$.

Lemma 13. For $p$ an odd prime, $k \mid p-1, k \nmid \frac{1}{2}(p-1)$, we have

$$
\sum_{m=1}^{(p-1) / 2}\left(\frac{m}{p}\right)_{k} \neq 0
$$

Equivalently, if $\chi$ is a character to the modulus $p$ with $\chi(-1)=-1$, then $\sum_{m=1}^{(p-1) / 2} \chi(m) \neq 0$.

Proof. Define

$$
e_{p}(m)=e^{2 \pi m / p} \quad \text { and } \quad \tau=\sum_{m=1}^{p-1}\left(\frac{m}{p}\right)_{k} e_{p}(m) .
$$

Then, by the proof on p. 22 of $[2],|\tau|=p^{1 / 2}$. From

$$
\sum_{m=1}^{p-1}\left(\frac{m}{p}\right)_{k} e_{p}(m n)=\sum_{m^{\prime}=1}^{p-1}\left(\frac{m^{\prime}}{p}\right)_{k}\left(\frac{n}{p}\right)_{k}^{-1} e_{p}\left(m^{\prime}\right)=\overline{\left(\frac{n}{p}\right)_{k}} \tau,
$$

we deduce that

$$
\overline{\left(\frac{n}{p}\right)_{k}}=\frac{1}{\tau} \sum_{m=1}^{p-1}\left(\frac{m}{p}\right)_{k} e_{p}(m n)
$$

Define

$$
L(s)=\sum_{n=0}^{\infty}\left(\frac{n}{p}\right)_{k} n^{-s} .
$$

As is well known (see [2], Chapter 1), $L(1) \neq 0$. The lemma is trivial for $k \leq 2$, so only the simpler proof of $L(1) \neq 0$ for $k>2$ is needed. From the 
Euler product,

Since

$$
\begin{aligned}
L(1) & =\left(1-\frac{1}{2}\left(\frac{2}{p}\right)_{k}\right)^{-1} \sum_{\substack{n=1 \\
n \text { odd }}}^{\infty}\left(\frac{n}{p}\right)_{k} n^{-1} \\
& =\left(1-\frac{1}{2}\left(\frac{2}{p}\right)_{k}\right)^{-1} \frac{1}{\bar{\tau}} \sum_{m=1}^{p-1} \overline{\left(\frac{m}{p}\right)_{k}} \sum_{\substack{n=1 \\
n \text { odd }}}^{\infty} \frac{e_{p}(-m n)}{n} .
\end{aligned}
$$

$$
\sum_{\substack{n=1 \\ n \text { odd }}}^{\infty} \frac{1}{n} z^{n}=\frac{1}{2} \log (1+z)-\frac{1}{2} \log (1-z)
$$

for $|z|=1, z \neq \pm 1$, where the logarithms have their principal values, we get

$$
\begin{aligned}
L(1)=\left(1-\frac{1}{2}\left(\frac{2}{p}\right)_{k}\right)^{-1} \frac{1}{\bar{\tau}}\left\{\sum _ { 0 < \theta < \pi } \left(-\frac{1}{2} \log \left(2 \sin \frac{1}{2} \theta\right)-\frac{1}{4}(\theta-\pi) i\right.\right. \\
\left.+\frac{1}{2} \log \left(2 \sin \frac{1}{2}(\theta+\pi)\right)+\frac{1}{4} \theta i\right) \overline{\left(\frac{m}{p}\right)_{k}} \\
+\ldots+\sum_{\pi<\theta<2 \pi}\left(-\frac{1}{2} \log \left(2 \sin \frac{1}{2} \theta\right)-\frac{1}{4}(\theta-\pi) i\right. \\
\left.\left.+\frac{1}{2} \log \left(2 \sin \frac{1}{2}(\theta-\pi)\right)+\frac{1}{4}(\theta-2 \pi) i\right) \overline{\left(\frac{m}{p}\right)_{k}}\right\},
\end{aligned}
$$

where $\theta=2 \pi-2 \pi m / p$. Hence

$$
\begin{aligned}
& L(1)=\left(1-\frac{1}{2}\left(\frac{2}{p}\right)_{k}\right)^{-1} \\
& \times \frac{1}{\bar{\tau}}\left\{\sum_{m<p / 2} \overline{\left(\frac{m}{p}\right)_{k}}\left(-\frac{1}{2} \pi i\right)+\sum_{m>p / 2} \overline{\left(\frac{m}{p}\right)_{k}} R_{1}(m)+\sum_{m<p / 2} \overline{\left(\frac{m}{p}\right)_{k}} R_{2}(m)\right\},
\end{aligned}
$$

where $R_{1}(m)=\frac{1}{2} \log \left(\cot \frac{1}{2} \theta\right)$ and $R_{2}(m)=\frac{1}{2} \log \left(-\cot \frac{1}{2} \theta\right)$, so that, for $m<p / 2$,

$$
R_{1}(p-m)=\frac{1}{2} \log \left(\cot \frac{1}{2}(2 \pi-\theta)\right)=\frac{1}{2} \log \left(-\cot \frac{1}{2} \theta\right) .
$$

Now

$$
\overline{\left(\frac{-m}{p}\right)_{k}}=\left(\frac{-1}{p}\right)_{k} \overline{\left(\frac{m}{p}\right)_{k}}=-\overline{\left(\frac{m}{p}\right)_{k}}
$$

since $k \nmid \frac{1}{2}(p-1)$, and so

$$
\sum_{m<p / 2} \overline{\left(\frac{m}{p}\right)_{k}} R_{2}(m)=-\sum_{m>p / 2} \overline{\left(\frac{m}{p}\right)_{k}} R_{1}(m) .
$$

The result follows from $L(1) \neq 0$. 
Lastly in this section, suppose $r$ is even and $l$ is an odd prime. The determinant is zero if and only if $\sum_{i=1}^{p-1} h_{i}=0$ or $h_{1}=h_{2}=\ldots=h_{p-1}$. Note first that

$$
\sum_{i=1}^{p-1} h_{i}=\left(p^{r}-p\right) f_{r}(1) \neq 0,
$$

so suppose that $h_{1}=h_{2}=\ldots=h_{p-1}$. Then

$$
h_{1}=\frac{p^{r}-p}{p-1} f_{r}(1) \quad \text { and } \quad c_{i}=c_{j}, \quad 1 \leq i, j \leq p-1,
$$

and so

$$
f_{1}=h_{1}+f_{r}(1)=\frac{1}{p}\left(c_{0}-c_{1}\right)=-\frac{1}{p} \sum_{j=0}^{r-1}\left(\begin{array}{l}
r \\
j
\end{array}\right) f_{j}(1) p^{j}
$$

Hence

$$
\frac{p^{r}-1}{p-1} f_{r}(1)=-\frac{1}{p} \sum_{j=1}^{r-1}\left(\begin{array}{l}
r \\
j
\end{array}\right) f_{j}(1)\left(p^{j}-1\right) .
$$

Now $2^{a} \|(2 l+1)^{2^{b}}-1$ where $a=b+1+c(b)$ where

$$
c(b)= \begin{cases}0, & b=0, \\ v_{2}(l+1), & b>0 .\end{cases}
$$

Suppose $2^{k} \| r$. Then

$$
2^{k+1+c(k)-2} \| \frac{p^{r}-1}{p-1} f_{r}(1) .
$$

If $2^{m} \| j$, then $\left(\begin{array}{l}r \\ j\end{array}\right) f_{j}(1)\left(p^{j}-1\right)$ is divisible by $2^{k-m-1+m+1+c(m)}=2^{k+c(m)}$. For $m=0$, the only non-zero term has $j=1$ and is $r(p-1) / 2 p$, which has 2 -adic valuation $k$. Hence the right-hand side of (13) has 2-adic valuation $k$. Hence $c(k)=1$, so that $v_{2}(l+1)=1$ and $l \equiv 1(\bmod 4)$. Thus the conjecture is valid for $l \equiv 3(\bmod 4)$ for $r$ even. Also a $p$-adic analysis can be used to show the conjecture is correct if $d=p$ is a regular prime.

The case $d=p^{2}$. Let $p$ be an odd prime, set $d=p^{2}, k=\frac{1}{2}\left(p^{2}-p\right)$, $l=\frac{1}{2}(p-1)$ and let $g$ be a primitive root modulo $d$. Define $I=$ $\left\{1, g, g^{2}, \ldots, g^{k-1}, p, p g, p g^{2}, \ldots, p g^{l-1}\right\}$, when for $r$ even the matrix of Conjecture 2 becomes

$$
\left(\begin{array}{ccc|c} 
& & & B \\
& A & & \vdots \\
& & & B \\
\hline B & \cdots & B & 0
\end{array}\right)
$$


and for $r$ odd,

$$
\left(\begin{array}{ccccc|c} 
& & & & & B^{\prime} \\
& & & & -B^{\prime} \\
& & & & B^{\prime} \\
& & & & \vdots \\
& & & & & B^{\prime} \\
\hline B^{\prime} & -B^{\prime} & B^{\prime} & \cdots & B^{\prime} & 0
\end{array}\right),
$$

where

$$
\begin{aligned}
A & =\left(\begin{array}{cccc}
h_{1} & h_{g} & \cdots & h_{g^{k-1}} \\
h_{g} & h_{g^{2}} & \cdots & h_{1} \\
\vdots & \vdots & & \vdots \\
h_{g^{k-1}} & h_{1} & \cdots & h_{g^{-2}}
\end{array}\right), \\
B & =\left(\begin{array}{cccc}
h_{p} & h_{p g} & \cdots & h_{p g^{l-1}} \\
h_{p g} & h_{p g^{2}} & \cdots & h_{p} \\
\vdots & \vdots & & \vdots \\
h_{g^{l-1}} & h_{p} & \cdots & h_{p g^{-2}}
\end{array}\right),
\end{aligned}
$$

and $A^{\prime}$ and $B^{\prime}$ are formed respectively from $A$ and $B$ by reversing the sign of all elements below the trailing diagonal. Note that $B$ (or $B^{\prime}$ ) is the relevant matrix for the case $d=p$, so, since the determinants of the above matrices are divisible by $(\operatorname{det} B)^{2}$ and $\left(\operatorname{det} B^{\prime}\right)^{2}$ respectively, if the conjecture fails for $d=p$ it fails for $d=p^{2}$ also.

Examples of $S \sim T$. Let $S=\left\{s_{1}, s_{2}, \ldots\right\}$ and $T=\left\{t_{1}, t_{2}, \ldots\right\}$.

(a) If $N(S)=N_{1}(S) \leq 1$ and $N(T)=N_{1}(T) \leq 1$ then $S \sim T$. If the conditions of (a) fail then $N=N(S)=N(T)$.

(b) $N=2: S \sim T$ if and only if $s_{1} s_{2}=t_{1} t_{2}$ and no number divides exactly three of $s_{1}, s_{2}, t_{1}, t_{2}$.

(c) $N=3: S \sim T$ if and only if $S=T$ or $\sum_{i=1}^{3} s_{i}=\sum_{i=1}^{3} t_{i}, \prod_{i=1}^{3} s_{i}=$ $\prod_{i=1}^{3} t_{i}$ and there exists $c$ such that $(1 / c) S$ and $(1 / c) T$ are each multisets of pairwise coprime elements. An example of this is

$$
S=\{m+1,2 m-1, m(2 m+1)\}, \quad T=\{m, 2 m+1,(m+1)(2 m-1)\},
$$

for $m \geq 3$ and $m \neq \equiv 2(\bmod 3)$.

(d) $N=4: S \sim T$ if and only if $S=T$ or $\prod_{i=1}^{4} s_{i}=\prod_{i=1}^{4} t_{i}, \sum_{i=1}^{4} s_{i}=$ $\sum_{i=1}^{4} t_{i}, \sum_{i=1}^{4} s_{i}^{2}=\sum_{i=1}^{4} t_{i}^{2}$, and there exists $c$ such that $(1 / c) S$ and $(1 / c) T$ are coprime multisets of elements such that no number divides exactly three elements of $S$ or exactly three elements of $T$. Further, if $d$ divides, without loss of generality, $s_{1}, s_{2}, t_{1}, t_{2}$ and $d$ does not divide $s_{3}, s_{4}, t_{3}, t_{4}$ then, without loss of generality, $s_{3} \equiv t_{3}(\bmod d)$ and $s_{4} \equiv t_{4}(\bmod d)$ and $s_{1} s_{2}=t_{1} t_{2}$. 
Proof. From $\left(1-\varrho^{-s_{3}}\right)\left(1-\varrho^{-s_{4}}\right)=\left(1-\varrho^{-t_{3}}\right)\left(1-\varrho^{-t_{4}}\right)$ and $d \mid s_{3}+$ $s_{4}-t_{3}-t_{4}$, we have $\varrho^{-s_{3}}+\varrho^{-s_{4}}=\varrho^{-t_{3}}+\varrho^{-t_{4}}$, whence, without loss of generality, $\varrho^{-s_{3}}=\varrho^{-t_{3}}$ and $\varrho^{-s_{4}}=\varrho^{-t_{4}}$. An example is

$$
\begin{aligned}
& S=\{m+6,6 m+13, m(m+8),(m+2)(6 m+1)\}, \\
& T=\{m+8,6 m+1,(m+2)(m+6), m(6 m+13)\},
\end{aligned}
$$

where $m$ does not belong to certain residue classes modulo $2,3,5,7,11,13$, 23,47 , so that $S$ and $T$ are sets of pairwise coprime elements. If we relax one of the conditions $m \not \equiv 2,4(\bmod 5)$ and $m \not \equiv 1,6(\bmod 7)$, however, we still have a solution. For example, if $m \equiv 2(\bmod 5)$, then $5 \mid s_{2}, s_{3}, t_{1}, t_{4}$, $s_{2} s_{3}=t_{1} t_{4}, s_{1} \equiv t_{2} \equiv 3(\bmod 5)$ and $s_{4} \equiv t_{3} \equiv 2(\bmod 5)$.

(e) Elements of $S$ (and therefore $T$ ) coprime in pairs and $N \geq 3$ :

$S \sim T \Leftrightarrow \prod s=\prod t, \sum s=\sum t$ and $\sum s^{2 k}=\sum t^{2 k}$ for $2 k \leq N-2$.

Conjecture 3. There are solutions of $S \sim T$ with $S \neq T$ in case (e) for all $N \geq 3$.

A more general theorem. Theorem 3 easily generalises to

TheOREM 6. $P_{S}(n)-P_{T}(n)=O\left(n^{j}\right)$ if and only if

(i) $N_{d}(S)=N_{d}(T)$ whenever $\max \left(N_{d}(S), N_{d}(T)\right) \geq j+2$,

(ii) $d_{S, r}=d_{T, r}$ for $1 \leq r \leq N_{d}-(j+2)$ for all $d$ with $N_{d} \geq j+3$,

(iii) $\prod_{s \in S, d \mid s} s=\prod_{t \in T, d \mid t} t$ whenever $N_{d} \geq j+2$,

(iv) $\prod_{s, d \nmid s}\left(1-\varrho^{-s}\right)=\prod_{t, d \nmid t}\left(1-\varrho^{-t}\right)$ whenever $N_{d} \geq j+2$.

An equivalent reformulation of the original problem. Expressing $S \sim T$ in terms of generating functions leads to the following result.

LEMMA 14. $S \sim T$ if and only if

$$
Q(x)=\left(1-x^{Z}\right)\left(\prod_{s \in S}\left(1-x^{s}\right)^{-1}-\prod_{t \in T}\left(1-x^{t}\right)^{-1}\right)
$$

is a polynomial of degree at most $Z-1$.

P r o o f. For $Q(x)$ a polynomial of degree $Z-1, Q(x) /\left(1-x^{Z}\right)$ is a general power series whose coefficients are periodic of period $Z$, and we know that if $P_{S}(n)-P_{T}(n)$ is bounded then it is of period $Z$.

LEMma 15. $S \sim T$ if and only if $\prod_{s \in S}\left(1-x^{s}\right)-\prod_{t \in T}\left(1-x^{t}\right)$ has a zero of order at least $2 N_{d}-1$ at $x=\varrho$, for $\varrho$ a primitive $d$-th root of unity, where $N_{d}=N_{d}(S)=N_{d}(T) \geq 2$.

Proof. This follows from Lemma 14 together with (i) of Theorem 3. 
The difficulties of this approach become apparent when one calculates

$$
\begin{aligned}
\frac{d^{r}}{d x^{r}} \prod_{i}\left(1-x^{s_{i}}\right)= & \sum_{\substack{a_{1}+\ldots+a_{N}=r\\
}}(-1)^{\Sigma_{a_{i}>0} 1} \\
& \times\left[\prod_{i}\left(\begin{array}{c}
s_{i} \\
a_{i}
\end{array}\right) a_{i} !\right] x^{\Sigma_{a_{i}>0} s_{i}-r} \prod_{\substack{s_{i} \\
a_{i}=0}}\left(1-x^{s_{i}}\right)\left(\begin{array}{c}
r \\
a_{1}, \ldots, a_{N}
\end{array}\right) .
\end{aligned}
$$

Partitions into an odd or even number of parts. With the notation as in the introduction, $Q_{S}(n)=P_{S}^{(0)}(n)-P_{S}^{(1)}(n)$ has the generating function

$$
\sum Q_{S}(n) x^{n}=\prod_{s \in S}\left(1+x^{s}\right)^{-1},
$$

and hence, by Theorem 2,

$$
\begin{aligned}
Q_{S}(n)=\sum_{d} & \sum_{\substack{\varrho \\
\varrho \text { primitive } \\
d \text { th root of } 1}}\left\{\prod_{s \equiv d / 2} s \prod_{s \neq d / 2}\left(1+\varrho^{-s}\right)\right\}^{-1} \\
& \times \sum_{j=0}^{M_{d}-1} \frac{1}{j !} \sum_{\substack{\text { partitions } \\
\text { of } M_{d}-1-j}} \prod_{r=1}^{M_{d}-1-j} \frac{e_{S, r}^{h_{r}}}{(r ! r)^{h_{r}} h_{r} !} \varrho^{n} n^{j}
\end{aligned}
$$

where $M_{d}=M_{d}(S)=\#\left\{s \in S: s \equiv \frac{1}{2} d(\bmod d)\right\}$ for $d$ even and $M_{d}=0$ for $d$ odd, and

$$
\sum_{r} \frac{e_{S, r} x^{r}}{r !}=\sum_{s} \frac{s x}{1+\varrho^{s} e^{s x}} .
$$

We note, in passing, the following result for which the full force of Theorem 2 is not required, but merely that $Q_{S}(n)$ is bounded if and only if $M_{d} \leq 1$ for all $d$, which follows from the non-singularity of the Vandermonde determinant as before.

THEOREM 7. The difference between the number of partitions of $n$ into an even number of parts from the multiset $S$ and the number of partitions into an odd number of parts from $S$ is bounded if and only if the 2-adic valuations of the elements of $S$ are distinct.

Proof. If $2^{r} \| d$, then $M_{d} \leq M_{2^{r}}=\#\left\{s \in S: 2^{r-1} \| s\right\}$, so if the 2-adic valuations of $S$ are distinct, then $M_{d} \leq 1$. Conversely, if $M_{2^{r+1}} \leq 1$, then at most one element of $S$ has 2-adic valuation $r$.

We consider when the two equations

$$
P_{S}^{(0)}(n)-P_{T}^{(0)}(n)=O(1) \quad \text { and } \quad P_{S}^{(1)}(n)-P_{T}^{(1)}(n)=O(1)
$$


hold. These are equivalent to

$$
P_{S}(n)-P_{T}(n)=O(1) \quad \text { and } \quad Q_{S}(n)-Q_{T}(n)=O(1) .
$$

Theorem 8. $Q_{S}(n)-Q_{T}(n)=O(1)$ if and only if

(i) $M_{d}(S)=M_{d}(T)$ whenever $\max \left(M_{d}(S), M_{d}(T)\right) \geq 2$,

(ii) $e_{S, r}=e_{T, r}$ for all $r$ with $1 \leq r \leq M_{d}-2$ for all $d$ with $M_{d}=$ $M_{d}(S)=M_{d}(T) \geq 3$,

(iii) $\prod_{s \equiv d / 2} s \prod_{s \neq d / 2}\left(1+\varrho^{-s}\right)=\prod_{t \equiv d / 2} t \prod_{t \not \equiv d / 2}\left(1+\varrho^{-t}\right)$ whenever $M_{d} \geq 2$.

Proof. Imitate the proof of Theorem 3.

Theorem 9. $P_{S}^{(0)}(n)-P_{T}^{(0)}(n)=O(1)$ and $P_{S}^{(1)}(n)-P_{T}^{(1)}(n)=O(1)$ if and only if

(a) $N_{d}(S)=N_{d}(T)$ if either is at least 2 , and $M_{d}(S)=M_{d}(T)$ if either is at least 2 ,

(b) $\sum_{a=0}^{d-1} f_{r}\left(\varrho^{a}\right) \sigma_{a}=0$ for all $r$ such that $1 \leq r \leq \max \left(N_{d}, M_{d}\right)-2$,

(c) $\prod_{s, d \nmid s}\left(1-\varrho^{-s}\right)=\prod_{t, d \nmid t}\left(1-\varrho^{-t}\right)$ where $\varrho$ is a primitive $d$-th root of unity for $\max \left(N_{d}, M_{d}\right) \geq 2$,

(d) $\prod_{s, d \mid s} s=\prod_{t, d \mid t} t$ if $\max \left(N_{d}, M_{d}\right) \geq 2$.

Pr o of. The conditions concerning $N_{d}(S)$ and $N_{d}(T)$ are the conditions of Theorem 3. Suppose these and the conditions of Theorem 8 hold. From (i) of Theorem 8, we have (a). Now observe that $M_{d}(S)=N_{d / 2}(S)-N_{d}(S)$, so that if $M_{d}(S)=M_{d}(T) \geq 2$, then $N_{d / 2}(S) \geq 2$, so $N_{d / 2}(S)=N_{d / 2}(T)$ and hence $N_{d}(S)=N_{d}(T)$. Note that $M_{l}(S) \geq M_{d}(S)$ when $l \mid d$ and $d / l$ is odd, so if $M_{d} \geq 2$, then $M_{l} \geq 2$ and hence $N_{l}(S)=N_{l}(T)$. If, on the other hand, $l \mid d$ and $d / l$ is even, then $l \mid \frac{1}{2} d$ so $N_{l}(S) \geq N_{d / 2}(S) \geq 2$ and so $N_{l}(S)=N_{l}(T)$ for all $l \mid d$. Hence, by Lemma $7, N_{d, a}(S)=N_{d, a}(T)$, and by an argument analogous to that in the proof of Theorem 3, we obtain

$$
\text { ( } \alpha) \prod_{s \equiv \frac{1}{2} d} s=\prod_{t \equiv \frac{1}{2} d} t \quad \text { and } \quad(\beta) \prod_{s \neq \frac{1}{2} d}\left(1+\varrho^{-s}\right)=\prod_{t \neq \frac{1}{2} d}\left(1+\varrho^{-t}\right) .
$$

Since $N_{d / 2}(S)=N_{d / 2}(T) \geq 2$, we have $\prod_{d / 2 \mid s} s=\prod_{d / 2 \mid t} t$ and combining this with $(\alpha)$ gives $\prod_{d \mid s} s=\prod_{d \mid t} t$. Again since $N_{d / 2} \geq 2$, we have $\prod_{d / 2 \nmid s}\left(1-\varrho^{-2 s}\right)=\prod_{d / 2 \nmid t}\left(1-\varrho^{-2 t}\right)$ and combining with $(\beta)$ gives

$$
\frac{\prod_{d / 2 \nmid s}\left(1-\varrho^{-s}\right)}{\prod_{d \mid s}\left(1+\varrho^{-s}\right)}=\frac{\prod_{d / 2 \nmid t}\left(1-\varrho^{-t}\right)}{\prod_{d \mid t}\left(1+\varrho^{-t}\right)}
$$

and hence $\prod_{d / 2 \nmid s}\left(1-\varrho^{-s}\right)=\prod_{d / 2 \nmid t}\left(1-\varrho^{-t}\right)$, since $N_{d}(S)=N_{d}(T)$. Condition (c) follows on multiplying by the factor $\prod_{s \equiv d / 2}\left(1-\varrho^{-s}\right)=2^{M_{d}}$ on either side. 
Finally, we deal with the equation $e_{S, r}-e_{T, r}=\sum_{a=0}^{d-1} f_{r}\left(-\varrho^{a}\right) \sigma_{a}=0$. Observe that

$$
f_{r}(\mu)+f_{r}(-\mu)=2^{r} f_{r}\left(\mu^{2}\right)
$$

so we have

$$
\sum_{a=0}^{d-1} 2^{r} f_{r}\left(\varrho^{2 a}\right) \sigma_{a}-\sum_{a=0}^{d-1} f_{r}\left(\varrho^{a}\right) \sigma_{a}=0 .
$$

However, $\left(\varrho^{2}\right)^{d / 2}=1$ and $N_{d / 2} \geq 2$, so $\sum_{a=0}^{d-1} 2^{r} f_{r}\left(\varrho^{2 a}\right) \sigma_{a}=0$, and hence $\sum_{a=0}^{d-1} f_{r}\left(\varrho^{a}\right) \sigma_{a}=0$ as required.

Conversely, it is clear from the above argument that, given (a) to (d), one could write down an explicit periodic formula for each of $P_{S}(n)-P_{T}(n)$ and $Q_{S}(n)-Q_{T}(n)$, if one wished.

Note that if $l \mid d$ then either $M_{l} \geq M_{d}$ or $N_{l} \geq M_{d}$, so in (b) we have $\sum_{a=0}^{d-1} f_{r}\left(\varrho^{a}\right) \sigma_{a}=0$ for all $\varrho$ such that $\varrho^{d}=1$. It follows that all the results and conjectures related to the conditions of Theorem 3 have obvious analogues related to Theorem 9 .

\section{References}

[1] G. E. Andrews, Partitions, originally: Encyclopedia of Mathematics, Vol. 2, Addison-Wesley, reprinted by Cambridge University Press, 1976.

[2] H. Daven port, Multiplicative Number Theory, 2nd ed., Springer, 1980.

[3] J. W. L. Glaisher, Formulae for partitions into given elements, derived from Sylvester's theorem, Quart. J. Math. 40 (1909), 275-348.

[4] J. J. Sylvester, On subinvariants, i.e. semi-invariants to binary quantics of an unlimited order: Excursus on rational functions and partitions, Amer. J. Math. 5 (1882), 119-136.

[5] E. M. Wright, Partitions into $k$ parts, Math. Ann. 142 (1961), 311-316.

DEPARTMENT OF MATHEMATICS

UNIVERSITY OF NOTTINGHAM

UNIVERSITY PARK, NOTTINGHAM

NG7 2RD, U.K.

Received on 7.2.1991

and in revised form on 2.4.1992 